\title{
Bagging, Random Subspace Method and Biding
}

\author{
Satoshi Shirai, Mineichi Kudo, and Atsuyoshi Nakamura \\ Division of Computer Science \\ Graduate School of Information Science and Technology \\ Hokkaido University \\ Kita-14, Nishi-9, Kita-ku, Sapporo 060-0814, Japan \\ \{satoshi,mine, atsu\}@main. ist .hokudai .ac.jp \\ http://prml.main.ist.hokudai.ac.jp
}

\begin{abstract}
In recent years, many approaches for achieving high performance by combining some classifiers have been proposed. We exploit many random replicates of samples in the bagging, and randomly chosen feature subsets in the random subspace method. In this paper, we introduce a method for selecting both samples and features at the same time and demonstrate the effectiveness of the method. This method includes a parametric bagging and a parametric random subspace method as special cases. In some experiments, this method and the parametric random subspace method showed the best performance.
\end{abstract}

\section{Introduction}

Many classifiers have been proposed for pattern recognition, including plug-in Bayes classifiers, nearest neighbors, support vector machines, etc. It is well known that no classifier keeps the best position for every problem, so that we have to choose one of them depending on the problem at hand. When the number of training samples is small, an appropriate classifier with a complexity suitable for the problem size must be chosen. One attractive approach is to combine several classifiers to bring stability over them. This approach is called classifier fusion and has been widely studied [1|2|3|45]. Each such classifier is called a component classifier. This approach aims at decreasing the generalized error by producing many weak classifiers in one way or another from a provided training dataset and by consolidating them in majority voting (the case of classification) or averaging (the case of regressive prediction).

Two methods have been widely used: bagging [1] and random subspace method [4. In bagging, many different training sets are generated by resampling and fed to a base classifier (a designing algorithm). On the other hand, in the random subspace method, many different feature subsets are randomly chosen for producing component classifiers. As a natural extension, simultaneous selection of sample and feature subsets has been studied [6] and has been shown to often outperform one-side selections. 
The advantageous effect of classifier fusion has been analyzed in different ways. In the case of regressive prediction, it has been revealed that independent, hopefully negatively-correlated, component classifiers are effective. Even in the case of classification, with some different definitions of correlation, use of negatively-correlated component classifiers is known to be effective 678. On the other hand, diversity of component classifiers 91011 is often a useful measure to estimate the performance. Typically, higher diversity of component classifiers means better performance in fusion.

In this paper, we focus on resampling rates of bagging and the random subspace method. Examining possible resampling rates in a systematical way is useful for revealing the potential abilities of bagging and the random subspace method in detail. In addition, we propose simultaneous selection of sample and feature subsets with optimized resampling rates.

\section{Formulation of Classifier Fusion}

Producing different component classifiers from a single training sample set by resampling of samples or by reselection of features or both is typical in classifier fusion. Let us divide a training set into form and contents. Here, the form is denoted by

$$
U_{N \times M}=T_{N} \times F_{M},
$$

where $T_{N}$ is the index set of samples, $T_{N}=\{1,2, \ldots, N\}$, and $F_{M}$ is the index set of features, $F_{M}=\{1,2, \ldots, M\}$. The contents are specified by a sampling (an observation) function $x: U_{N \times M} \rightarrow R$ with $(i, j) \mapsto x_{i j}$. Hereafter, $U$ and the relatives are considered along with the contents $\left\{x_{i j}\right\}$ unless no confusion occurs.

\subsection{Number of Distinct Elements by Resampling}

In some cases, it is better to consider the set of distinct samples instead of the bootstrap sample $T^{*}$ itself. Indeed, duplication of samples does not cause any change in the rule of nearest neighbors and support vector machines without a soft margin.

When $N \alpha(0 \leq \alpha \leq 1)$ elements are randomly and uniformly selected from $N$ elements with replacement, the expected size $n=E \hat{n}$ of distinct elements is given by

$$
n=\left(1-\left(1-\frac{1}{N}\right)^{N \alpha}\right) N \simeq\left(1-e^{-\alpha}\right) N .
$$

Thus, $\theta=n / N=0.63$ for $\alpha=1$ (bagging case).

\subsection{Bagging}

In bagging, one resampling selects $T_{N}^{*}$ of $N$ samples from $T_{N}$ of $\mathrm{N}$ samples, but some samples in $T_{N}^{*}$ are duplicated. The average size $n$ of distinct samples is 
$n=0.63 N$. As a result, on average, we have the form $U_{n \times M}=T_{n} \times F_{M}$. The bagging method is a majority vote of predicted classes by $\phi\left(T_{N}^{* 1}\right), \phi\left(T_{N}^{* 2}\right), \ldots, \phi\left(T_{N}^{* B}\right)$, where $\phi$ is a base classifier and $T_{N}^{* 1}, T_{N}^{* 2}, \ldots, T_{N}^{* B}$ are the B bootstrapped samples. The corresponding $T_{n_{1}}^{1}, T_{n_{2}}^{2}, \ldots, T_{n_{B}}^{B}$ of distinct samples are different in size, but the basic performance for fixed $\theta_{S}^{B}=0.63$ and $n=0.63 N$ can be analyzed.

\subsection{Random Subspace Method}

On the other hand, in the random subspace method [4], $F_{M / 2}^{*}$ of $M / 2$ distinct features are typically selected randomly from $F_{M}$ of $M$ features. As a result, we have the form $U_{N \times M / 2}$ with $\theta_{F}^{R}=0.5$ and $m=0.5 M$. Similarly to bagging, we use B bootstrapped features $F_{M / 2}^{1}, F_{M / 2}^{2}, \ldots, F_{M / 2}^{B}$ for designing component classifiers.

\subsection{Parametric Bagging and Random Subspace Method}

Let us analyze the performance of above two aggregating methods of component classifiers produced by random sampling with/without replacement. To make clear the difference in which direction sampling is made, we use $\theta_{S}$ for samples and $\theta_{F}$ for features. Then we have

$$
\begin{aligned}
& U_{\theta_{S} N \times M}=T_{\theta_{S} N} \times F_{M}:(\text { parametric }) \text { bagging, } \\
& U_{N \times \theta_{F} M}=T_{N} \times F_{\theta_{F} M}:(\text { parametric }) \text { random subspace method } .
\end{aligned}
$$

The question "what are the best values of $\theta_{S}$ and $\theta_{F}$ ?" naturally arises. To examine the relationship between $\theta_{S}\left(\theta_{F}\right)$ and classification performance, we examined 10 real-world datasets. They are UCI datasets [12] that have wore 10 featuresand consist of continuous features only. The classification rate was estimated by 10 -fold cross validation. In the following, the bagging is simulated by $\theta_{S}=0.63$ and the random subspace method by $\theta_{F}=0.5$. In this case, a certain subset of size $n(m)$ is chosen from a set of size $N(M)$ with a probability of $1 /\left(\begin{array}{l}N \\ n\end{array}\right)\left(1 /\left(\begin{array}{l}M \\ m\end{array}\right)\right)$. Here, $\theta_{S}\left(\theta_{F}\right)$ was changed in the range of 0.1 to 1.0 by step 0.1 . The base classifier was the nearest-neighbor and $B=100$. The results are shown in Table 1 and Table 2. In bagging, the parameter tuning did not greatly improve the performance. However, it did improve the performance when $\theta_{S}$ was low (for example, in heart-statlog and waveform-5000, the best value of $\theta_{S}$ is $10 \%)$. This is an astonishing result. This result implies the possibility of sparse sampling. Note that the nearest neighbor does not change its rule if $\theta_{S}>0.5$, such as $\theta_{S}^{B}=0.63$ for bagging, because the true nearest neighbor of a given sample appears over a half of $T_{n}^{1}, T_{n}^{2}, \ldots, T_{n}^{B}$ with $n=\theta_{S} N$. The classification rates using the parametric random subspace method were improved at $3 \%$ on average from those using the original random subspace method. The optimal value of $\theta_{F}^{*}$ ranges from 0.2 to 0.7 but is mostly around 0.3 . That is, the empirical selection $\theta_{F}=0.5$ of a typical random subspace method is a little larger than the best choice. 
Table 1. Recognition rates in bagging (Bag) with 1-NN. Here, $\theta_{S}^{*}$ is the sampling ratio bringing the best performance.

\begin{tabular}{ccccccc}
\hline Dataname & \#class & \#sample 1-NN $\operatorname{Bag}\left(\theta_{S}^{B}=63 \%\right)$ & P-Bag $\left(\theta_{S}^{*} \%\right)$ & P-Bag/Bag \\
\hline heart-statlog & 2 & 270 & 59.62 & 59.62 & $68.14(10)$ & 1.148 \\
ionosphere & 2 & 351 & 86.92 & 86.92 & $87.76(50)$ & 1.009 \\
optdigits & 10 & 5620 & 98.75 & 98.75 & $98.84(30)$ & 1.001 \\
page-blocks & 5 & 5473 & 95.41 & 95.43 & $95.88(30)$ & 1.004 \\
segment & 7 & 2310 & 96.49 & 96.49 & $96.49(60-100)$ & 1.000 \\
sonar & 2 & 208 & 83.64 & 83.64 & $83.64(60-100)$ & 1.000 \\
spambase & 2 & 4601 & 82.41 & 82.37 & $82.78(40)$ & 1.004 \\
vehicle & 4 & 846 & 65.71 & 65.71 & $66.42(50)$ & 1.010 \\
waveform-5000 & 3 & 5000 & 76.58 & 76.68 & $84.52(10)$ & 1.102 \\
wine & 3 & 178 & 74.73 & 74.73 & $75.26(30)$ & 1.007 \\
\hline Average & & & 82.03 & 82.03 & $83.97(41.0)$ & 1.028 \\
\hline
\end{tabular}

Table 2. Recognition rates in the random subspace method (RS) with 1-NN.Here, $\theta_{F}^{*}$ is the sampling ratio bringing the best performance

\begin{tabular}{cccccc}
\hline Dataname & \#feature SVM RS $\left(\theta_{F}^{R}=50 \%\right)$ & $\mathrm{P}-\mathrm{RS}\left(\theta_{F}^{*} \%\right)$ & $\mathrm{P}-\mathrm{RS} / \mathrm{RS}$ \\
\hline heart-statlog & 13 & 59.62 & 66.67 & $73.33(40)$ & 1.100 \\
ionosphere & 34 & 86.92 & 90.61 & $94.02(20)$ & 1.037 \\
optdigits & 64 & 98.75 & 99.02 & $99.05(70)$ & 1.000 \\
page-blocks & 10 & 95.41 & 95.52 & $95.70(60)$ & 1.002 \\
segment & 19 & 96.49 & 97.74 & $97.74(50)$ & 1.000 \\
sonar & 60 & 83.64 & 85.07 & $89.90(20)$ & 1.056 \\
spambase & 57 & 82.41 & 91.72 & $93.21(30)$ & 1.016 \\
vehicle & 18 & 65.71 & 69.14 & $71.14(20)$ & 1.029 \\
waveform-5000 & 40 & 76.58 & 84.60 & $84.82(30)$ & 1.002 \\
wine & 13 & 74.73 & 88.20 & $96.07(30)$ & 1.089 \\
\hline Average & & 82.03 & 86.83 & $89.50(37.0)$ & 1.033 \\
\hline
\end{tabular}

\subsection{Biding}

Choice of the best pair $\left(\theta_{S}, \theta_{F}\right)^{*}$ is clearly more effective than a single choice of best $\theta_{S}^{*}$ or $\theta_{F}^{*}$. Therefore, we consider simultaneous selection of $\left(\theta_{S}, \theta_{F}\right)$ and call the aggregating method using the selected parameter pair "Biding" (abbreviation of BIDirectional aggregatING). In biding, a component classifier has a form

$$
U_{\theta_{S} N \times \theta_{F} M}=T_{\theta_{S} N} \times F_{\theta_{F} M}: \text { biding. }
$$

Parametric bagging $(\mathrm{P}-\mathrm{Bag})$ corresponds to the case of $\left(\theta_{S}, 1.0\right)$ and the parametric random subspace method (P-RS) corresponds to the case of $\left(1.0, \theta_{F}\right)$. The functional relation between performance and $\left(\theta_{S}, \theta_{F}\right)$ is shown in Fig 1 concretely for heart-statlog and segment datasets. From Fig 1 , we see the effectiveness of simultaneous selection. We conducted the same experiment with $\left(\theta_{S}, \theta_{F}\right)$. The performance is shown in Table 3. Biding is naturally the best among all three 


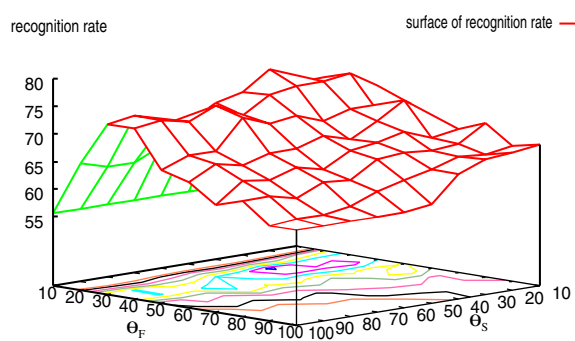

(a) heart-statlog

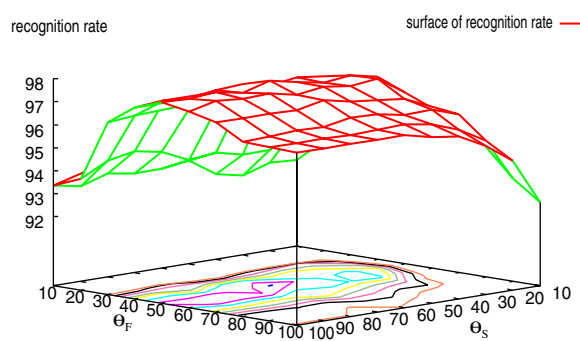

(b) segment

Fig. 1. Recognition rate over $\left(\theta_{S}, \theta_{F}\right)$ : (a) the best pair $\left(\theta_{S}, \theta_{F}\right)^{*}=(40,30)$ in heartstatlog, (b) the best pair $\left(\theta_{S}, \theta_{F}\right)^{*}=(60,50)$ in segment

Table 3. Comparison of parametric bagging (P-Bag), parametric random subspace (P-RS) method and biding

\begin{tabular}{cccccc}
\hline Dataname & P-Bag $\left(\theta_{S}^{*} \%\right)$ & P-RS $\left(\theta_{F}^{*} \%\right)$ & Biding $\left(\theta_{S} \%, \theta_{F} \%\right)^{*}$ & Biding/P-Bag Biding/P-RS \\
\hline heart-statlog & $68.14(10)$ & $73.33(40)$ & $78.51(40,30)$ & 1.152 & 1.070 \\
ionosphere & $87.76(50)$ & $94.02(20)$ & $94.02(100,20)$ & 1.071 & 1.000 \\
optdigits & $98.84(30)$ & $99.05(70)$ & $99.06(60,80)$ & 1.002 & 1.000 \\
page-blocks & $95.88(30)$ & $95.70(60)$ & $96.08(40,90)$ & 1.002 & 1.004 \\
segment & $96.49(60-100)$ & $97.74(50)$ & $97.92(60,50)$ & 1.015 & 1.002 \\
sonar & $83.64(60-100)$ & $89.90(20)$ & $89.92(90,20)$ & 1.075 & 1.000 \\
spambase & $82.78(40)$ & $93.21(30)$ & $93.21(100,30)$ & 1.125 & 1.000 \\
vehicle & $66.42(50)$ & $71.14(20)$ & $71.50(60,30)$ & 1.076 & 1.005 \\
waveform-5000 & $84.52(10)$ & $84.82(30)$ & $85.84(20.60)$ & 1.015 & 1.012 \\
wine & $75.26(30)$ & $96.07(30)$ & $97.18(40,20)$ & 1.291 & 1.011 \\
\hline Average & 83.97 & 89.50 & 90.32 & 1.082 & 1.010 \\
\hline
\end{tabular}

variants including itself. The improvement ratios to the parametric bagging and parametric random subspace method with the optimal parameters were 1.08 and 1.01 , respectively.

Table 3 also shows the difference between $\left(\theta_{S}, \theta_{F}\right)^{*}$ and $\left(\theta_{S}^{*}, \theta_{F}^{*}\right)$. From Fig 2 , we see that $\left(\theta_{S}, \theta_{F}\right)^{*}$ tends to be located at the right-hand or upper-right of $\left(\theta_{S}^{*}, \theta_{F}^{*}\right)$. This is natural because either $\theta_{S}$ or $\theta_{F}$ is fixed to one when $\theta_{S}^{*}$ or $\theta_{F}^{*}$ is calculated. Combining results shown in Table 1, Table 2, and Table 3 , biding improved the original (but simulated) bagging at ratio $0.3-31.7 \%$ and the random subspace method at ratio $0.2-2.3 \%$.

\section{Estimation of $\left(\theta_{S}, \theta_{F}\right) *$ by Optimization}

For practical usage, we have to estimate the optimal parameters in biding and its two variants. It is natural to use a validation dataset cut from the training set. The problem is the cost of calculation. It costs the number of $\left(\theta_{S}, \theta_{F}\right)$ pairs to be 


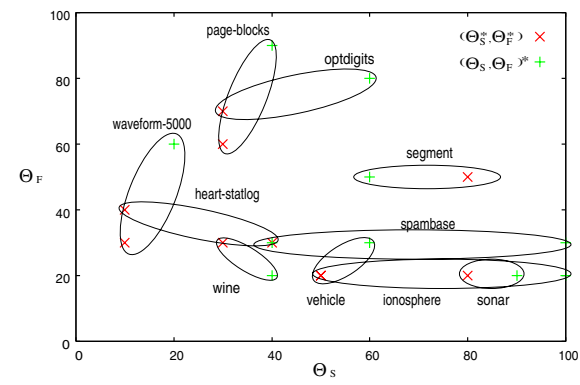

Fig. 2. Point diagram of $\left(\theta_{S}^{*}, \theta_{F}^{*}\right)$ and $\left(\theta_{S}, \theta_{F}\right)^{*}$. An ellipsoid encloses the same dataset.

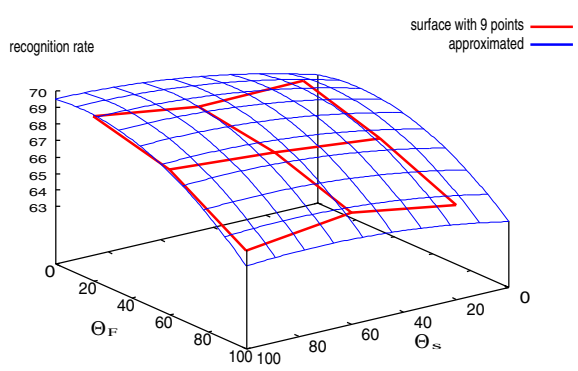

Fig. 3. Quadratic approximation by nine points

examined multiplied by the cost of one-time validation test and by the number $\mathrm{B}$ of bootstrapping. It is therefore crucial to reduce the size of the candidate set of $\left(\theta_{S}, \theta_{F}\right)$ 's.

To do this, assuming smoothness of the target function (see Fig 11), we approximate it by a quadratic function that is estimated surrounding eight points and the center point (Fig 3 . Then we narrow the search region by a factor of $1 / 4$ for once. By three times of narrowing, we find an estimation of $\left(\theta_{S}, \theta_{F}\right)^{*}$ with 27 values of the target function on $[0.2,1.0] \times[0.2,1.0]$. For P-Bag and P-RS, we use 20 points in $[0.05,1.0]$ on each axis.

\section{Experiments}

We conducted some experiments to demonstrate the effectiveness of P-Bag, P-RS and biding. The base classifier is either of the nearest neighbor and the support vector machine (SVM) with a radial basis kernel, and $B=100$. The result of

Table 4. Recognition rate by SVM method

\begin{tabular}{ccccccc}
\hline Data & SVM & Bag & RS & P-Bag $\left(\theta_{S}^{*} \%\right)$ & P-RS $\left(\theta_{F}^{*} \%\right)$ & $\operatorname{Biding}\left(\left(\theta_{S}, \theta_{F}\right)^{*}\right)$ \\
\hline heart-statlog & 62.22 & 62.22 & 73.70 & $59.63(72.0)$ & $80.37(28.5)$ & $\mathbf{8 1 . 1 1}(33.2,21.0)$ \\
ionosphere & 93.46 & 92.60 & $\mathbf{9 4 . 0 4}$ & $92.31(83.5)$ & $92.90(64.5)$ & $92.61(58.2,49.6)$ \\
optdigits & 96.49 & 96.21 & $\mathbf{9 9 . 0 4}$ & $96.37(65.5)$ & $\mathbf{9 9 . 0 4}(50.0)$ & $98.81(58.3,38.8)$ \\
page-blocks & 92.42 & 92.51 & 94.06 & $92.44(80.5)$ & $\mathbf{9 4 . 4 6}(33.5)$ & $93.88(57.2,30.0)$ \\
segment & 92.51 & 92.38 & 96.74 & $92.55(93.5)$ & $96.71(43.0)$ & $\mathbf{9 7 . 1 0}(64.8,35.9)$ \\
sonar & 81.17 & 80.69 & 80.69 & $80.21(72.0)$ & $\mathbf{8 1 . 6 9}(55.5)$ & $76.68(51.0,44.7)$ \\
spambase & 86.61 & 86.79 & $\mathbf{9 3 . 7 4}$ & $86.55(65.0)$ & $93.68(43.0)$ & $93.33(51.8,41.9)$ \\
vehicle & 57.20 & 56.85 & 69.84 & $55.55(62.0)$ & $\mathbf{7 3 . 8 7}(29.0)$ & $72.21(34.8,21.9)$ \\
waveform-5000 & 83.08 & 85.08 & 85.78 & $\mathbf{8 6 . 5 2}(21.0)$ & $85.60(45.5)$ & $86.44(25.0,60.2)$ \\
wine & 70.72 & 69.64 & 89.87 & $69.64(61.5)$ & $96.57(32.5)$ & $\mathbf{9 7 . 7 1}(34.5,24.2)$ \\
\hline Average & 81.59 & 81.50 & 87.75 & $81.18(65.7)$ & $\mathbf{8 9 . 4 9}(42.5)$ & $88.98(46.9,36.8)$ \\
\hline
\end{tabular}


SVM is shown in Table 4. We also obtained the result of $1-\mathrm{NN}$, but it is not shown here for economizing the space, but the tendency was similar. We have tried to use SVM in the experiments of the previous section, but gave up due to its high computation cost.

In both 1-NN and SVM, P-RS was best for many cases and biding followed. This is mainly because the number 27 of search points in biding was insufficient for 2-dimensional search space, compared to 20 for 1-dimensional search space in the other two variants. Indeed, it is noted that when $\left(\theta_{S}, \theta_{F}\right)$ is chosen close to the optimal value in Table 3, the biding attained the best performance.

\section{Analysis of Classifier Fusion in Classification}

So far, many studies have been invoked to reveal when classifier fusion works best [1345]. The standard ways to connect the generalized error possibly reduced by classifier fusion with the characteristics of component classifiers can be divided into three: 1) bias and variance decomposition, 2) dependency analysis, that is, what degree the component classifiers are correlated to each other, and 3) diversity measures among component classifiers.

Let us first use a disagreement measure. The disagreement measure $D i s_{b, b^{\prime}}$ between component classifiers $\phi_{b}$ and $\phi_{b}^{\prime}$ is calculated by $N$ samples with $N_{1}$ outputs of both correct or both incorrect and $N_{0}$ outputs of one-sided correct such as

$$
D i s_{b, b^{\prime}}=\frac{N_{0}}{N_{0}+N_{1}}, \text { Dis }=\frac{1}{B(B-1)} \sum_{b} \sum_{b^{\prime} \neq b} D i s_{b, b^{\prime}} .
$$

The relationship between improvement rate and diversity is shown in Fig 4. In Fig 4. we notice that there is a positive correlation between them, and biding and random subspace method brought the largest diversity. Note that $\operatorname{Dis}_{b, b^{\prime}}$ is a post-event evaluation, that is, this value is available only with true labels. On the other hand, we can use this measure without such true labels if the number of classes is two.

Next, we state the relationship among above 1), 2) and 3) in a theoretical way. Let us assume that a classifier (strictly speaking, a design algorithm) $\phi$ produces an estimate $\hat{\eta}(x)$, with a training sample set, for the posterior density $\eta(x)=$ $P(Y=1 \mid X=x)$ in a two-class problem $(Y \in\{1,0\})$. Then the assignment by $\hat{\eta}$ is given by $\hat{y}(x)=\operatorname{sign}(\hat{\eta}(x)-1 / 2)$. Let $y_{B}(x)$ be the assignment by the Bayes classifier as $y_{B}(x)=\operatorname{sign}(\eta(x)-1 / 2)$.

According to Friedman [13, the bias-variance decomposition at a certain point $x$ for classification is given by a multiplicative form, unlike an additive form for regression, as

$$
P(\hat{y}(x) \neq Y)-P\left(y_{B}(x) \neq Y\right)=|2 \eta(x)-1| \operatorname{Erf}\left(\frac{-b(\eta(x), \mathrm{E} \hat{\eta}(x))}{\sqrt{\operatorname{Var} \hat{\eta}(x)}}\right),
$$

where $\operatorname{Erf}(x)$ is the error function defined by $\operatorname{Erf}(x)=1 / \sqrt{2 \pi} \int_{x}^{\infty} e^{-u^{2} / 2} d u$ and the boundary bias $b$ is given by

$$
b(\eta(x), \mathrm{E} \hat{\eta}(x))=\operatorname{sign}(\eta(x)-1 / 2)(1 / 2-\mathrm{E} \hat{\eta}(x)) .
$$




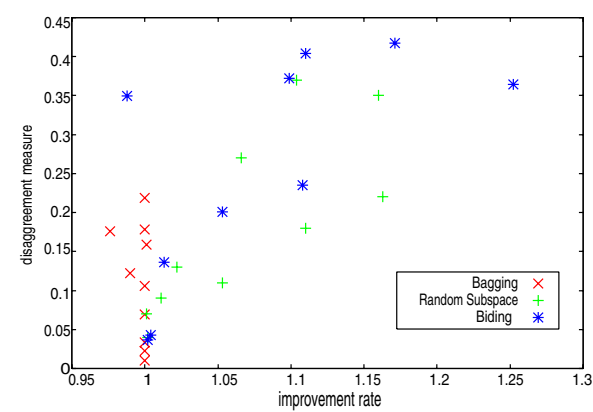

(a) $1-\mathrm{NN}$

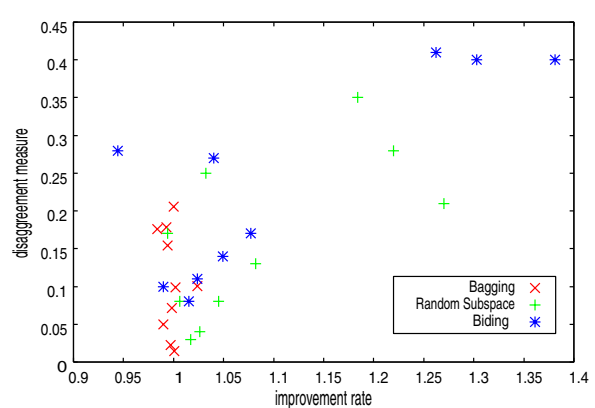

(b) SVM

Fig. 4. Relationship between improvement rate and diversity

That is, the generalized error $P(\hat{y}(x) \neq Y)$ at $x$ is expressed by the Bayes error plus a multiplicative decomposition of the boundary bias $b(\eta(x), \mathrm{E} \hat{\eta}(x))$ and the square-rooted variance $\sqrt{\operatorname{Var} \hat{\eta}(x)}$. Here, the distribution of $\hat{\eta}(x)$ is assumed to be a Gaussian to exploit the error function.

Eq. (2) implies that if the boundary bias is negative, hence the assignment is agreed between the Bayes classifier and $\phi$, then a smaller variance is effective for improving the performance, and, if not, a larger variance is effective for making $\operatorname{Erf}(x)$ approach $1 / 2$.

From (2), we have the total generalized error $\operatorname{Err}(\phi)$ as

$$
0 \leq \operatorname{Err}(\phi)-\operatorname{Err}_{\text {Bayes }}=\int|2 \eta(x)-1| \operatorname{Erf}\left(\frac{-b(\eta(x), \mathrm{E} \hat{\eta}(x))}{\sqrt{\operatorname{Var} \hat{\eta}(x)}}\right) p(x) d x .
$$

When aggregation is applied to $B$ component classifiers with

$$
\hat{y}_{1}, \hat{y}_{2}, \ldots, \hat{y}_{b}, \ldots, \hat{y}_{B}, \quad \forall b, \mathrm{E} \hat{y}_{b}=\mathrm{E} \hat{y},
$$

we have an aggregated estimate and assignment as

$$
\hat{\eta}_{A}=\frac{1}{B} \sum_{b} \hat{y}_{b}, \quad \hat{y}_{A}=\operatorname{sign}\left(\hat{\eta}_{A}-1 / 2\right) .
$$

Then,

$$
\begin{aligned}
\mathrm{E} \hat{\eta}_{A}=\mathrm{E} \hat{y}, \quad \operatorname{Var} \hat{\eta}_{A} & =\mathrm{E}\left\{\left(\frac{1}{B} \sum_{b} \hat{y}_{b}-\mathrm{E} \hat{y}\right)^{2}\right\} \\
& =\frac{1}{B} \operatorname{Var} \hat{y}+\frac{1}{B^{2}} \sum_{b} \sum_{b^{\prime} \neq b} \operatorname{Cov}\left(\hat{y}_{b}, \hat{y}_{b^{\prime}}\right),
\end{aligned}
$$

where

$$
\operatorname{Var} \hat{y}=\mathrm{E} \hat{y}(1-\mathrm{E} \hat{y}), \quad \operatorname{Cov}\left(\hat{y}_{b}, \hat{y}_{b^{\prime}}\right)=\mathrm{E}\left(\hat{y}_{b}-\mathrm{E} \hat{y}\right)\left(\hat{y}_{b^{\prime}}-\mathrm{E} \hat{y}\right) .
$$


That is, by aggregating, the variance is reduced by $1 / B$ and a positive (negative) correlation increases (decreases) the variance.

Here, we notice that

$$
\begin{aligned}
\mathrm{E}\left(\hat{y}_{b}-1 / 2\right)\left(\hat{y}_{b^{\prime}}-1 / 2\right) & =\mathrm{E}\left(\hat{y}_{b}-\mathrm{E} \hat{y}\right)\left(\hat{y}_{b^{\prime}}-\mathrm{E} \hat{y}\right)+(\mathrm{E} \hat{y}-1 / 2)^{2} \\
& =\operatorname{Cov}\left(\hat{y}_{b}, \hat{y}_{b^{\prime}}\right)+(\mathrm{E} \hat{y}-1 / 2)^{2} .
\end{aligned}
$$

Then, we can evaluate Eq. (5) as

$$
\begin{aligned}
& \operatorname{Var} \hat{\eta}_{A}=\frac{1}{B} \operatorname{Var} \hat{y}-\frac{1}{B^{2}} \sum_{b} \sum_{b^{\prime} \neq b} \mathrm{E}\left(\hat{y}_{b}-1 / 2\right)\left(1 / 2-\hat{y}_{b^{\prime}}\right) \\
& -\left(1-\frac{1}{B}\right)(\mathrm{E} \hat{y}-1 / 2)^{2} \text {. }
\end{aligned}
$$

That is, the variance is reduced if the degree of disagreement between $b$ and $b^{\prime}$ is (positively) high or if $\mathrm{E} \hat{y}$ is far from $1 / 2$, or both.

On the other hand, the disagreement measure Dis (1) is evaluated over $\left\{x_{i}\right\}$ as

$$
\begin{aligned}
\text { Dis }= & \frac{1}{B(B-1)} \sum_{b} \sum_{b^{\prime} \neq b} \frac{1}{N} \sum_{i=1}^{N}\left\{2\left(\hat{y}_{b}\left(x_{i}\right)-1 / 2\right)\left(1 / 2-\hat{y}_{b^{\prime}}\left(x_{i}\right)\right)+\frac{1}{2}\right\} \\
\rightarrow & \frac{2}{B(B-1)} \sum_{b} \sum_{b^{\prime} \neq b} \int\left(\hat{y}_{b}(x)-1 / 2\right)\left(1 / 2-\hat{y}_{b^{\prime}}(x)\right) p(x) d x+\frac{1}{2} \\
& \text { as } N \rightarrow \infty .
\end{aligned}
$$

Comparing Eqs. (7) and (8), we notice that Dis (10) shows to what degree the variance of the aggregated classifier is reduced on average as a sample estimation. The difference is that the expectation is taken for the training sets in (7) and is taken for $\{x\}$ in (8).

In total, (5), (7) and (8) reveal the relationship among the variance of an aggregating classifier, the correlation between component classifiers, and the disagreement between component classifiers. When the disagreement is high, then the correlation becomes negative and thus the variance of aggregation is reduced.

\section{Conclusion}

We have proposed a parametric bagging, a parametric random subspace method, and biding as a parent of them for classifier fusion. We confirmed that the parametric random subspace method and biding are both effective in most cases. We also found that 1) sparse sampling (a parametric bagging with a small sampling rate) may be useful for large datasets, 2) traditional random subspace method is reasonable but a little larger in the sampling rate, and 3) simultaneous random selection of samples and features is promising.

Among them, biding is the most powerful in theory. The main drawback of biding is the cost of estimating optimal parameters. In the experiments, a very 
simple technique for optimization was employed with only 27 function values. A more sophisticated optimization technique would be considered for improving the performance of biding.

\section{References}

1. Breiman, L.: Bagging predictors. Machine Learning Journal 2 24, 123-140 (1996)

2. Efron, B.: Bootstrap methods: Another look at the jackknife. Annals of Statistics 7, 1-26 (1979)

3. Freund, Y., Iyer, R., Shapire, E., Singer, Y.: An efficient boosting algorithm for combining preferences. The Jounal of Machine Learning Research 4, 933-969 (2003)

4. Ho, T.: The random subspace method for constructing decision forests. IEEE Trans. on Pattern Analysis and Machine Intelligence 20, 832-844 (1998)

5. Jiang, Y., Ling, J.J., Li, G., Dai, H., Zhou, Z.: Dependency Bagging. LNCS, pp. 491-500 (2005)

6. Skurichina, M., Duin, R.: Bagging, boosting and the random subspace method for linear classifiers. Pattern Analysis and Applications 5, 121-135 (2002)

7. Oh, S.: On the relationship between majority vote accuracy and dependency in multiple classifier systems. Pattern Recognition Letters 24, 359-363 (2003)

8. Ruta, D., Gabrys, B.: A theoretical analysis of the limits of majority voting errors for multiple classifier systems. Pattern Analysis and Applications 5, 333-350 (2002)

9. Brown, G., Wyatt, J., Harris, R., Yao, X.: Diversity creation methods: a survey and categorisation. Information Fusion 6, 5-20 (2005)

10. Kuncheva, L., Whitaker, C.: Measures of Diversity in Classifier Ensembles and Their Relationship with the Ensemble Accuracy. Machine Learning 51, 181-207 (2004)

11. Skalak, D.: The sources of increased accuracy for two proposed boosting algorithms. In: Proc. American Association for Artificial Intelligence, AAAI 1996, Integrating Multiple Learned Models workshop (1996)

12. Blake, C., Merz, C.: UCI repository of machine learning databases (1998), http://www.ics.uci.edu/ mlearn/mlrepository.html

13. Friedman, J.: On Bias, Variance, 0/1-Loss, and the Curse-of-Dimensionality. Data Mining and Knowledge Discovery 1, 55-77 (1997) 